\title{
Thulium Assisted Parametric Conversion from Near to Short Wave Infrared
}

\author{
Adrien Billat ${ }^{1}$, Steevy Cordette $^{1}$, Yu-Pei Tseng ${ }^{1}$ and Camille-Sophie Brès ${ }^{1}$ \\ 1. Photonic Systems Laboratory, Ecole Polytechnique Fédérale de Lausanne (EPFL) STI IEL, CH-1015 Lausanne, Switzerland \\ adrien.billat@epfl.ch
}

\begin{abstract}
We report an all-fiber continuous wave source, tunable between 1935-1980nm, based on parametric conversion combined with thulium amplification. More than $150 \mathrm{~mW}$ of power and $30 \mathrm{~dB}$ optical signal-to-noise ratio is obtained over the entire range.

OCIS codes: (190.4975) Parametric processes, (230.2285) Fiber devices and optical amplifiers, (060.4370) Nonlinear optics, fibers.
\end{abstract}

\section{Introduction}

The $2 \mu \mathrm{m}$ spectral region has gathered interest during the past years as these wavelengths are well suited for hollow core fiber communications [1], gas sensing or non-linear generation [2]. Most of these applications would highly benefit from an easily tunable, energy efficient and modulation capable source. Short wave infrared (SWIR) sources based on four wave mixing (FWM) in highly nonlinear fibers [3-5] have recently been demonstrated instituting them as strong candidates for modulation capable $2 \mu \mathrm{m}$ sources. It has however proven difficult to reach high level of powers while operating in the continuous wave regime. In this paper, we present the efficient generation in SWIR band based on the combination of parametric conversion and subsequent amplification of the newly generated idler in a Thulium doped fiber (TDF), both processes making use of the same C-band pump.

\section{Experimental setup}

The experimental setup is presented in figure 1 and consists in two cascaded stages: a fiber optics parametric amplifier (FOPA) and a Thulium doped fiber amplifier (TDFA). In the former, the continuous wave (CW) pump from an external cavity laser (ECL) at $1566 \mathrm{~nm}$ is phase-modulated with a pseudo-random bit sequence (PRBS) to suppress stimulated Brillouin scattering (SBS), and amplified by a high power Erbium doped fiber amplifier (EDFA) to obtain a power of $34.5 \mathrm{dBm}$ after filtering of the amplified stimulated emission (ASE). A signal wave from a tunable ECL in the O-band (1260 $\mathrm{nm}$ to $1360 \mathrm{~nm}$ ) is amplified by a semiconductor optical amplifier (SOA) to $16 \mathrm{dBm}$ and coupled with the pump through a 1310/1550 wavelength multiplexer (MUX1). Both are sent into $350 \mathrm{~m}$ of a highly nonlinear fiber (HNLF), with zero dispersion wavelength at $1569 \mathrm{~nm}$, a third order dispersion $\beta_{3}$ around $4.410^{-2} \mathrm{ps}^{3} \mathrm{~km}^{-1}$ and a negative $\beta_{4}$. The SBS return losses are monitored through a circulator. In the HNLF, FWM between the CW pump and signal results in the generation of a CW idler wave located between $1850 \mathrm{~nm}$ and $2070 \mathrm{~nm}$, depending on the wavelength of the O-band signal.

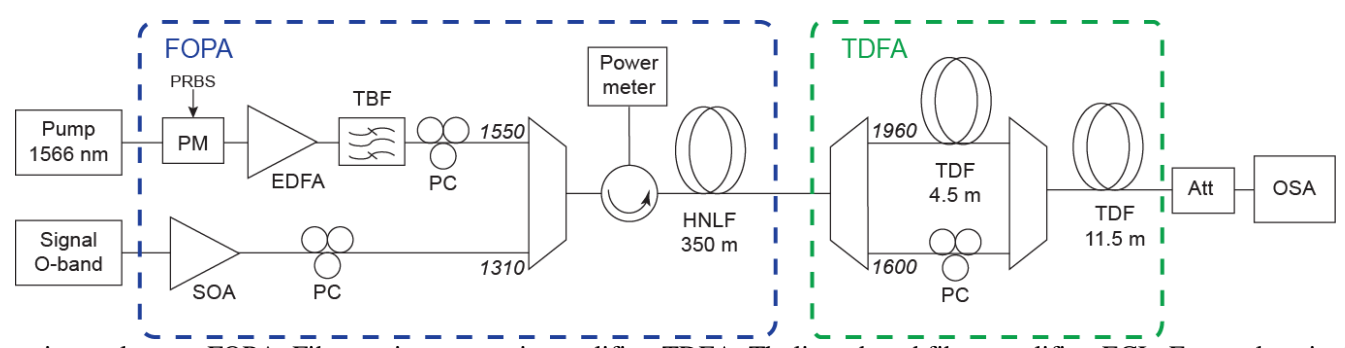

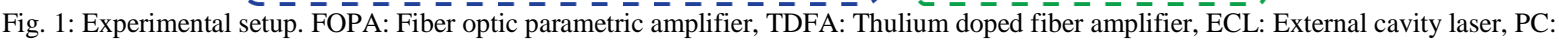
Polarization controller, PM: Phase modulator, SOA: Semiconductor optical amplifier, EDFA: Erbium doped fiber amplifier, TBF: Tunable band pass filter, HNLF: Highly nonlinear fiber, TDF: Thulium doped fiber, Att: C-band $20 \mathrm{~dB}$ attenuator, OSA: Optical spectrum analyzer.

The signal, pump and idler are then all directed to the TDFA stage. The idler and the signal are first separated from the pump by a suitable 1600/1960 multiplexer (MUX2). They subsequently flow through $4.5 \mathrm{~m}$ of Thulium doped fiber (TDF) and are recombined with the pump to be launched into another $11.5 \mathrm{~m}$ segment of TDF now pumped by the $1566 \mathrm{~nm}$ parametric pump. The $4.5 \mathrm{~m}$ TDF being pumped by the backward-propagating ASE generated in the $11.5 \mathrm{~m} \mathrm{TDF}$ [6], the idler is amplified in both stages whereas the signal undergoes absorption. The final spectra are recorded by an optical spectrum analyzer (OSA) after a $20 \mathrm{~dB}$ attenuator.

\section{Results and discussions}

The performance of the FOPA stage is first quantified by observing the HNLF output on the OSA. The signal wavelength was swept in the O-band and the superimposed spectra are shown in figure 2(a). Note that a C-band 
stated $20 \mathrm{~dB}$ attenuator was used before the OSA. The actual attenuation in the O-band and $2 \mu \mathrm{m}$ band of this element was characterized using the ASE of an SOA and a $2 \mu \mathrm{m}$ supercontinuum. This characterization was then used to calculate the conversion efficiency (CE) of the FOPA as plotted in figure 2(b). A clean conversion is observed with minimal parametric amplified noise in accordance with the expected negative $\beta_{4}$ FOPA pumped in the normal regime. The idler gain spectrum is broadened due to the dispersion fluctuations along the $350 \mathrm{~m}$ of HNLF [5]. A maximum CE of $-15 \mathrm{~dB}$ is achieved for an idler near $1950 \mathrm{~nm}$ with a $3 \mathrm{~dB}$ bandwidth close to $80 \mathrm{~nm}$. Idler up to $2071 \mathrm{~nm}$ were generated, limited by the tunable range of the O-band source. At the output of the HNLF, we measure $32 \mathrm{dBm}$ of remaining $1566 \mathrm{~nm}$ pump power which is used to directly pump the TDFA stage.

(a)

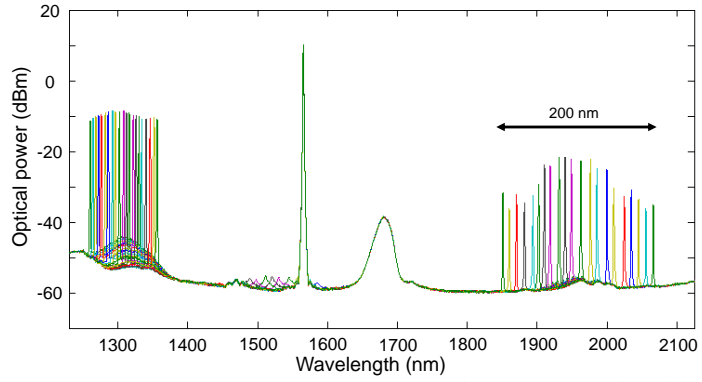

(b)

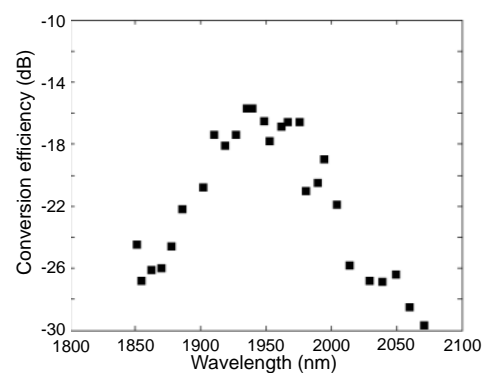

Fig. 2: (a) Superimposed spectra at output of FOPA stage for different O-band signals (resolution: $1 \mathrm{~nm}$ ). (b) Experimental CE.

The output of the FOPA-TDFA is shown in figure 3(a) for a $1307 \mathrm{~nm}$ input signal. Close to $27.5 \mathrm{~dB}$ of gain is observed on the $1953 \mathrm{~nm}$ generated idler while clear amplification in the $1900 \mathrm{~nm}-2000 \mathrm{~nm}$ band is detected. The original C-band pump is completely depleted by the Thulium stage while the original O-band signal is significantly absorbed, such that a simple bandpass filter in the $2 \mu \mathrm{m}$ band could be used to remove ASE without the burden of residual high power C- or O-band signals.

(a)

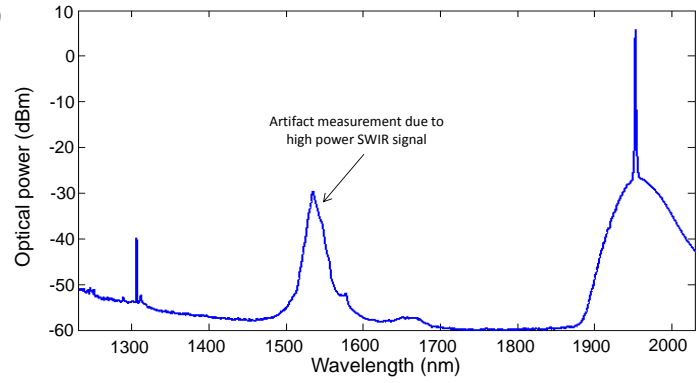

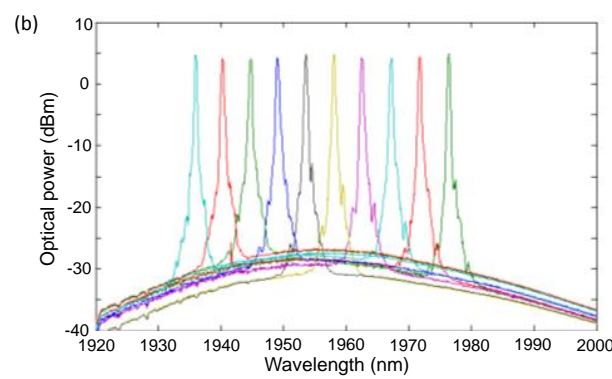

Fig 3: (a) Output spectrum of TDFA stage for a $1307 \mathrm{~nm}$ signal (resolution: $1 \mathrm{~nm}$ ). (b) Tunable range after TDFA stage (resolution: $0.5 \mathrm{~nm}$ ).

The tunability of the source is shown in figure 3 (c). A remarkably flat output over close to $45 \mathrm{~nm}$ is obtained. The CW output power of the thulium stage, taking into account the attenuation used for the monitoring on the OSA, is $22 \mathrm{dBm}$ for wavelengths between $1935 \mathrm{~nm}$ and $1980 \mathrm{~nm}$. Because of the bandwidth of the thulium amplifier, idler generated outside this band could not be efficiently amplified and are not shown in the figure. The optical signal-tonoise ratio (OSNR) is measured above $30 \mathrm{~dB}$ for the whole considered band. After appropriate filtering, this setup yields a $\mathrm{CW}$ tunable source in the $1.95 \mu \mathrm{m}$ range with an improved wall-plug efficiency compared to a simple FOPA, given that the same pump is used to first generate and then amplify the idler.

In conclusion, we demonstrated a $150 \mathrm{~mW}$ continuous wave SWIR source with $45 \mathrm{~nm}$ tunability. A TDF is used to enhance the output power of the FOPA while also acting as a low pass filter. The un-used FOPA pump power is efficiently recycled as the TDFA pump for an enhanced generation of SWIR light. Furthermore as the $2 \mu \mathrm{m}$ signal is initiated from a FOPA driven in the telecommunication band, such configuration can also be used for the transfer of one or multiple modulated seeds to the $2 \mu \mathrm{m}$ band.

This work is supported by the European Research Council under grant agreement ERC-2012-StG 306630-MATISSE. The authors would like to thank Sumitomo Electric Industries for providing the HNLF.

\section{References}

[1] M. N. Petrovich, et al., "Demonstration of amplified data transmission at $2 \mu \mathrm{m}$ in a low-loss wide bandwidth hollow core photonic bandgap fiber," Opt. Express 21, 28559-28569 (2013).

[2] Z. Li, et al., "All-fiber, ultra-wideband tunable laser at $2 \mu \mathrm{m}$," Opt. Lett. 38, 4739-4742 (2013).

[3] J.M. Chavez-Boggio, et al., "Tunable Parametric All-Fiber Short -Wavelength IR transmitter, " JLT, vol. 28(4), (2010).

[4] F. Gholami, et al., "10Gbps Parametric Short-Wave Infrared Transmitter," OFC conference proceedings (2011).

[5] F. Gholami, et al., "Phase-preserving parametric wavelength conversion to SWIR band in highly nonlinear dispersion stabilized fiber," Opt. Express 21, 11415-11424 (2013).

[6] Z. Li, et al., "Thulium-doped fiber amplifier for optical communications at $2 \mu \mathrm{m}, "$ Opt. Express 21, 9289 (2013). 